\title{
Effect of strain rate on yielding strength of a Zr-based bulk metallic glass
}

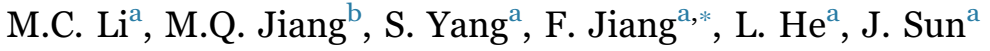 \\ a State Key Laboratory for Mechanical Behavior of Materials, Xi'an Jiaotong University, Xi'an 710049, China \\ ${ }^{\mathrm{b}}$ State Key Laboratory of Nonlinear Mechanics, Institute of Mechanics, Chinese Academy of Sciences, Beijing 100190, China
}

\section{A R T I C L E I N F O}

\section{Keywords:}

Bulk metallic glasses

Strain rate

Yielding strength

Free volume

Thermal softening

\begin{abstract}
A B S T R A C T
Uniaxial tension and compression experiments were performed on a typical $\mathrm{Zr}_{52.5} \mathrm{Cu}_{17.9} \mathrm{Ni}_{14.6} \mathrm{Al}_{10} \mathrm{Ti}_{5}\left(\mathrm{Vit}_{105}\right)$ bulk metallic glass over a wide range of strain rates at room temperature. It is found that the strain rate effect of the yielding strength will change from insensitive to negative with increasing strain rate above a critical value. This phenomenon can be quantitatively described by a modified cooperative-shear model of shear transformation zones that takes the adiabatic temperature rise into account. The model predicts well the present and other experimental data.
\end{abstract}

\section{Introduction}

Bulk metallic glasses (BMGs) have been long regarded as potential structural materials due to the unique properties in comparison with their crystalline counterparts. Their mechanical properties therefore have been extensively investigated [1-3]. A number of researchers have paid much attention to the strain rate sensitivity of strength in BMGs, which is important for engineering applications especially under dynamic loading. Ma et al. [4] and Zhang et al. [5] demonstrated a positive strain rate dependence of compressive yielding strength in Tibased BMGs, which was attributed to the different microstructure on the atomic scale. While Zheng et al. [6] and Li et al. [7] showed that the compressive yielding strength of Zr-based BMGs decreased with increasing strain rate and ascribed the negative strain rate dependence to the enhanced adiabatic heating in the shear bands under high strain rates. Moreover, it was observed that the strain rate dependence could change from insensitive to negative with increasing strain rate [8,9]. By performing quasi-static and dynamic tension, Mukai et al. [10] argued that the fracture strength (also yielding strength) of a $\mathrm{Pd}_{40} \mathrm{Ni}_{40} \mathrm{P}_{20}$ BMG was essentially independent on strain rate. Similar results had also been found by other researchers at strain rate from $10^{-5} \mathrm{~s}^{-1}$ to $10^{-1} \mathrm{~s}^{-1}$ in Zr-based BMGs [11,12]. Based on the nanoindentation method, Bhattacharyya et al. [13] presented a negative strain rate sensitivity in a Zr-based BMG. However, the work of Pan et al. [14] showed a positive rate sensitivity. Similar inconsistent results have been also reported [15-17].

In the present paper, we choose a typical Zr-based (Vit 105) BMG as the model material and perform the uniaxial loading (compression or tension) on it over a wide range of strain rates. The strain rate sensitivity of the measured yielding strength is discussed within a modified cooperative-shear model developed by Liu and Liu [18], which takes the adiabatic heating effect into account.

\section{Experiments}

Ingots with the nominal composition of $\mathrm{Zr}_{52.5} \mathrm{Cu}_{17.9} \mathrm{Ni}_{14.6} \mathrm{Al}_{10} \mathrm{Ti}_{5}$ (Vit 105) were prepared by arc-melting high purity $\mathrm{Zr}, \mathrm{Cu}, \mathrm{Ni}, \mathrm{Al}$, and $\mathrm{Ti}$ in a Ti-gettered argon atmosphere. The plates with dimensions of $40 \times 20 \times 2 \mathrm{~mm}^{3}$ were obtained using the drop casting method, and then they were machined into Dog-bone-shaped specimens with gauge dimensions of $6 \times 1 \times 0.8 \mathrm{~mm}^{3}$ for the tensile tests. Cylindrical specimens for compressive tests were cut from the as-cast cylindrical rods with a diameter of $\Phi 3 \mathrm{~mm}$, which were prepared via the copper-mold suctioncasting method. In order to eliminate the influence of sample size, the specimens for quasi-static and dynamic compression had a same aspect ratio of 2:1. Special care was taken to ensure that the two surfaces of the compression samples were parallel and orthogonal to the loading axis. The glassy state of all samples was verified by X-ray diffraction and high resolution transmission electron microscopy. Quasi-static tensile and compressive experiments were conducted on a computercontrolled SUNSCMT 5105 material testing machine with nominal strain rates of $2.0 \times 10^{-4} \mathrm{~s}^{-1}, 1.0 \times 10^{-3} \mathrm{~s}^{-1}$ and $1.0 \times 10^{-2} \mathrm{~s}^{-1}$ at room temperature. Tensile and compressive tests under dynamic strain rates of about $1.8 \times 10^{3} \mathrm{~s}^{-1}$ and $3.2 \times 10^{3} \mathrm{~s}^{-1}$ were performed on the Split Hopkinson Tensile Bar (SHTB) and Split Hopkinson Pressure Bar (SHPB), respectively. To guarantee an equilibrate state of stress and develop a uniform strain rate in the specimen up to the point of failure, a copper pulse-shaper was used between the impacting projectile and

\footnotetext{
* Corresponding author.

E-mail address: jiangfeng@mail.xjtu.edu.cn (F. Jiang).
} 


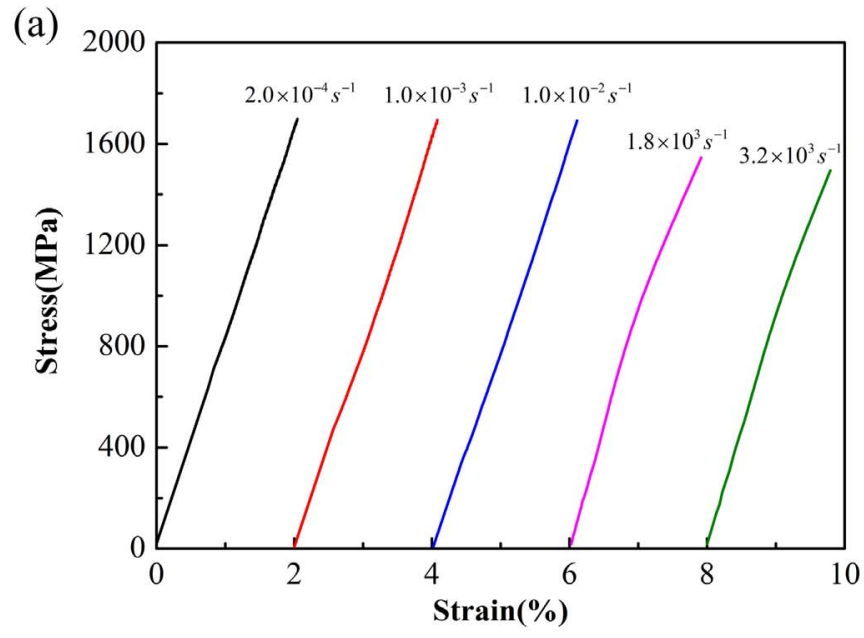

(b)

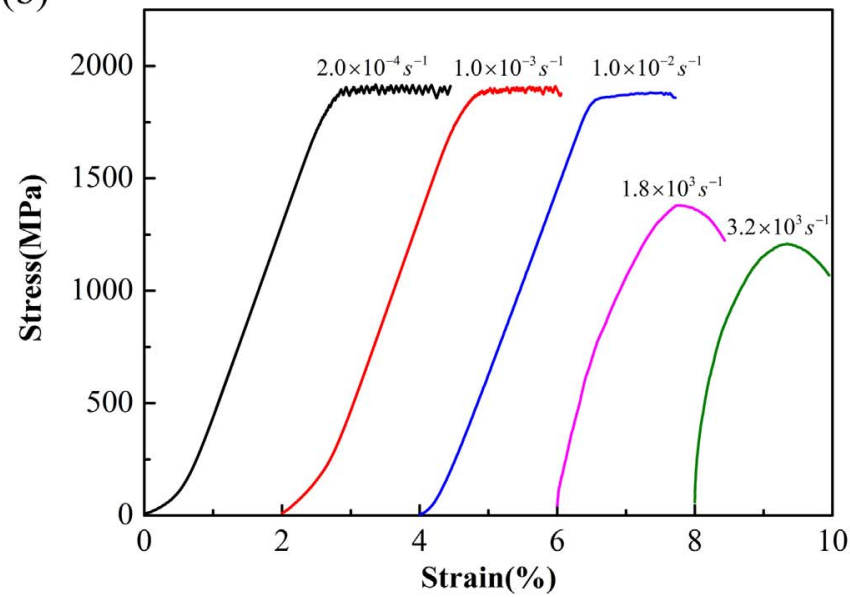

Fig. 1. Typical engineering stress-strain curves at room temperature under: (a) uniaxial tension; (b) uniaxial compression.

the input bar during both the dynamic tension and compression. In order to ensure the reliability of the experimental results, at least 6 samples were tested at each case. After tests, the morphologies of the fracture angle as well as the fracture surfaces were investigated by a high-resolution scanning electron microscopy (SEM: SU6600).

\section{Results}

Typical tensile engineering stress-strain curves of the Vit 105 under different strain rates at room temperature are shown in Fig. 1a (the dynamic curves were smoothed). All of them exhibit the linear elastic deformation and immediate fracture without any macroscopic plastic strain. Therefore, it is reasonable to consider that the tensile fracture strength is the same as the tensile yielding strength of the BMG. Here, it must be addressed that the bending of the curves in the dynamic cases does not mean any plasticity, but is due to the inevitably unstable signal of the measured strain. Apparently, increasing the strain rate from $2.0 \times 10^{-4} \mathrm{~s}^{-1}$ to $1.0 \times 10^{-2} \mathrm{~s}^{-1}$ does not lead to an obvious variation of yielding strength. However, when the strain rate increases to $1.8 \times 10^{3} \mathrm{~s}^{-1}$ or $3.2 \times 10^{3} \mathrm{~s}^{-1}$, the yielding strength shows an apparent decrease comparing to that of $2.0 \times 10^{-4} \mathrm{~s}^{-1}$. In order to reveal the deformation mechanism of the Vit 105 glass under different strain rates, the fractured samples were examined by the SEM. Fig. 2 presents the macroscopic fracture modes and the microscopic fracture surface morphologies of these samples. Apparently, all of these samples fracture along a single shear band. In addition, the fracture angles have a slight increase when the strain rate increases by 7 orders of magnitude, however, all of these angles fall in the common range for BMGs in uniaxial tensions [19-22]. Fig. 2a and b show that the fracture surfaces under low strain rates are relatively smooth. However, the samples tested under dynamic cases break with an irregularly fracture plane, i.e., there are a lot of gaps in the vicinity of the edge (Fig. 2c). The fracture surface morphologies corresponding to Fig. 2a-c are shown in Fig. $2 \mathrm{~d}-\mathrm{f}$ at low magnification. Obviously, the fracture surface becomes rougher with increasing strain rate. Especially, for the samples broke under $3.2 \times 10^{3} \mathrm{~s}^{-1}$, there are many pits and bumps on the fracture surface. This might result from the randomly distribute free volume sites, which have nearly no time to move and rearrange for the formation of a regular shear band [23-25]. This indicates that the existence of the free volume may have an effect on the yielding strength of the BMG. With further magnifying the areas circled by the dashed rectangles in Fig. $2 \mathrm{~d}-\mathrm{f}$, similar micro-scale smooth cores and vein patterns are observed on the shear fracture surfaces, as shown in Fig. 2g-i. However, after a careful comparison between Fig. $2 g$ and i, it can be found that the amount of the molten droplets on the dynamically-fractured surface is much more than that on the quasistatic one. Combining the yielding strength with the correspondingly fracture surface morphologies, we can find that the lower yielding strength corresponds to a heavier molten behavior. This demonstrates that the thermal effect should play an important role in the yielding behavior of the BMG under dynamic cases.

As for the uniaxial compressions, the engineering stress-strain curves under different strain rates are presented in Fig. 1b (the curves obtained from dynamic tests were also smoothed). It can be found that all of these samples have a linearly elastic response followed by an apparently yielding behavior. Similar to the uniaxial tension conditions, the compressive yielding strength under quasi-static strain rates is also insensitive to the strain rate. However, the yielding strength has a more significant reduction under dynamic compression than tension, which finally results in a lower yielding strength under dynamic compression than tension. Fig. $1 \mathrm{~b}$ also shows that there is a relatively large plastic flow under quasi-static conditions, while the samples fractured under dynamic cases with nearly no plastic flow but a sudden failure. To have a deeper understanding of the cases responsible for this unique trend, the fracture angles of the samples deformed under uniaxial compressions are shown in Fig. 3a-c. Obviously, all of these samples also fracture along a single shear band. At the same time, the fracture angles have a slight deviation from $45^{\circ}$ due to the normal stress effect. The fracture surface morphologies corresponding to Fig. 3a-c were also observed by SME, as shown in Fig. $3 \mathrm{~d}-\mathrm{f}$. Accordingly, we can find that the failure plane of the samples fractured under quasi-static compression is relatively smooth (Fig. 3d), while that fractured under dynamic cases is rugged (Fig. 3f). This is similar to the observations under the uniaxial tension, which stems from the insufficient time for the diffusion and the rearrangement of the randomly distribute free volume sites. The corresponding magnified details of the areas circled by the dashed rectangles in Fig. $3 \mathrm{~d}-\mathrm{f}$ are shown in Fig. 3g-i. Vein-like patterns can be observed on the fracture surface of all the samples, demonstrating the typical compressive shear fracture [12,26-28]. However, the fracture surface morphologies are different from each other when a close attention is paid to Fig. $3 \mathrm{~g}-\mathrm{i}$. Apparently, the fracture surface under $2.0 \times 10^{-4} \mathrm{~s}^{-1}$ is mainly characterized by vein-like patterns, indicating the occurrence of a local viscous flow. For $1.0 \times 10^{-2} \mathrm{~s}^{-1}$, the vein-like patterns are blocked by a number of smooth bands, which might stem from the thermal molten and the combination of the vein-like patterns. When the strain rate increases to $3.2 \times 10^{3} \mathrm{~s}^{-1}$, there are a large number of molten droplets distributed randomly on the shear fracture surface, which implies a heavier thermal effect under dynamic cases. Furthermore, we can find a more serious molten behavior under dynamic compression than tension according to Figs. $2 \mathbf{i}$ and $3 \mathbf{i}$, which should contribute to the lower dynamic yielding strength under compression than tension. 

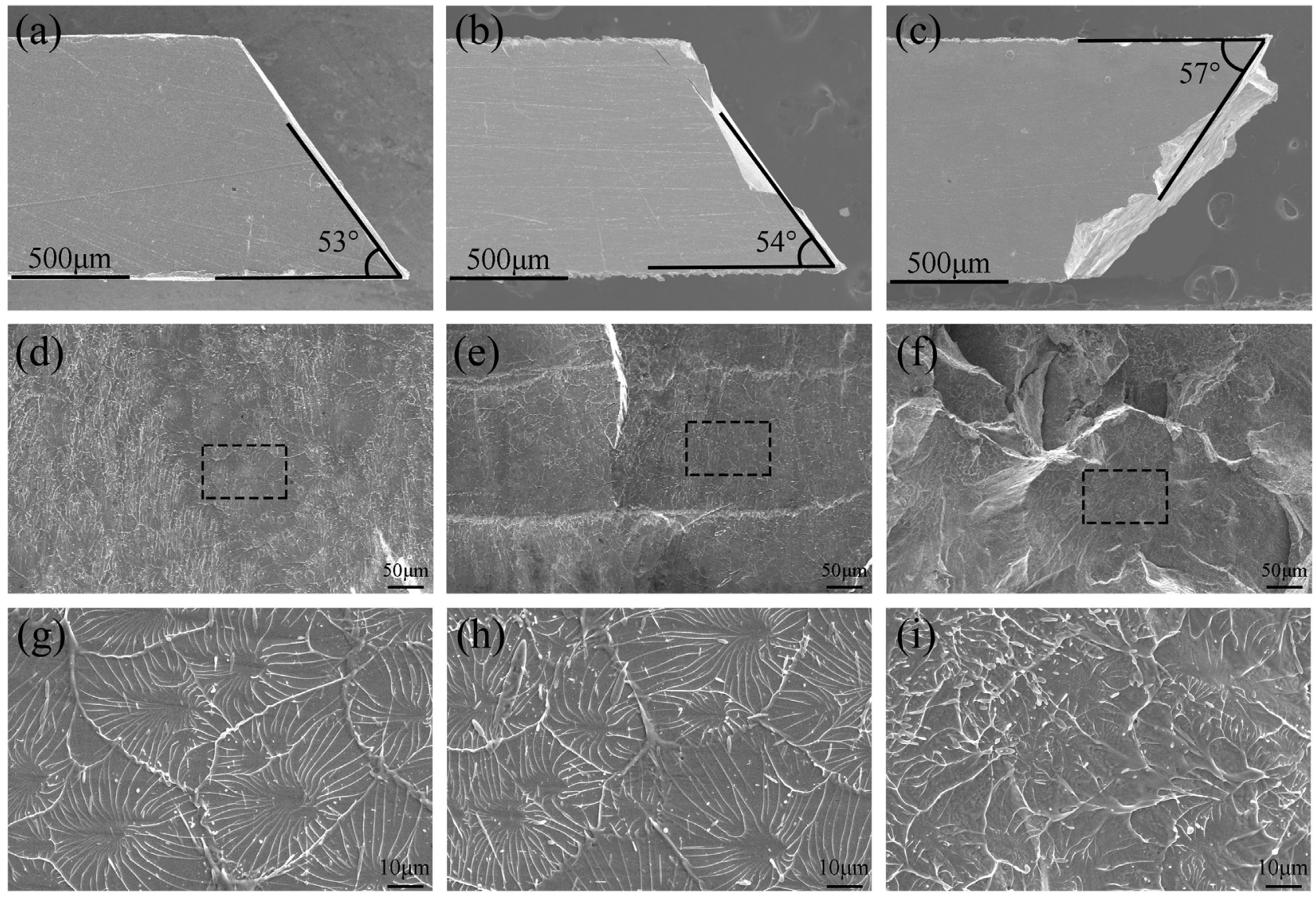

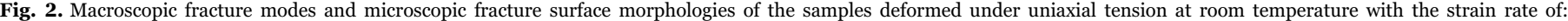
$2.0 \times 10^{-4} \mathrm{~s}^{-1}$ : (a), (d), (g); $1.0 \times 10^{-2} \mathrm{~s}^{-1}$ : (b), (e), (h); $3.2 \times 10^{3} \mathrm{~s}^{-1}$ : (c), (f), (i).

\section{Discussion}

The observations presented in the previous section unambiguously show that the yielding strength of the Zr-based BMG is firstly insensitive and then negative to the increasing strain rate. Moreover, there is a lower yielding strength under dynamic compression than tension, which overturns the yielding strength difference between compression and tension under quasi-static condition. In the following, the underlying mechanism related to these phenomena will be discussed.

It is widely accepted that shear transformation zone (STZ) is the embryo of localized shear event in BMGs at atomic-scale, and the yielding of BMGs corresponds to the unsteady propagation of a large number of STZs [29-32]. The cooperative shear model of STZs developed by Johnson and Samwer [30] gives an universal law for the shear yielding strength of BMGs as:

$\tau_{C T}=\tau_{C 0}-\tau_{C 0}\left[(k / \beta) \ln \left(\omega_{0} / C \dot{\gamma}\right)\left(G_{0 T} / G_{0 T_{g}}\right)\right]^{2 / 3}\left(T / T_{g}\right)^{2 / 3}$.

where $\tau_{C T}$ and $\tau_{C 0}$ are the shear yielding strength at a finite temperature $T$ and $T=0$, respectively. $C$ and $\beta$ are constant, $\dot{\gamma}$ is shear strain rate. $T_{g}$ is the glass transition temperature, $k$ and $\omega_{0}$ are the Boltzmann constant and attempt frequency $\left(\sim 10^{13} \mathrm{~Hz}\right)$, respectively. $G_{0 T} / G_{0 T_{g}}$ is the ratio of the shear modulus at finite temperature $T$ and at $T_{g}$, which incorporates the weak dependence of shear modulus on the thermal expansion of a fixed glass configuration. Eq. (1) indicates that the shear yielding stress of the BMG has a positive dependence on the shear strain rate and a negative dependence on the finite temperature. At the meantime, the more serious molten behavior under dynamic cases (Figs. 2 and 3) implies that the finite temperature may be dependent on the applied strain rate. Therefore, it is necessary to clarify the relationship between the applied strain rate and the finite temperature. Based on cooperative shear model of STZs [30,33,34], Liu and Liu [18] deduced an equation of the temperature rise around an emerging STZ as:

$\vartheta=\frac{\zeta \Omega \eta \dot{\gamma}^{2}}{8 \pi \kappa r_{0}}$.

where $\vartheta$ is the temperature rise relative to room temperature. $\zeta$ is a correction factor and should be taken as $\zeta=3$ [30]. $\Omega$ is the STZ volume. $\eta$ and $\kappa$ are the viscosity and the thermal conductivity near the contiguous STZ, respectively. $r_{0}$ is the characteristic radial distance of the heat source, which should be approximately equal to the radius of the STZ. Consequently, the temperature around an emerging STZ along with increasing shear strain rate should be taken as:

$T=T_{r}+\frac{\zeta \Omega \eta \dot{\gamma}^{2}}{8 \pi \kappa r_{0}}$

where $T_{r}$ is room temperature and is taken to be $293 \mathrm{~K}$.

According to Eq. (3), it can be seen that the temperature around an emerging STZ has a positive dependence on the shear strain rate. Therefore, there will be a more serious thermal effect during the deformation under a higher strain rate. This is agreement with the more serious melting behavior on the dynamic fracture surfaces (Figs. 2 and 3). As shown in Figs. 2 and 3, the fracture angles under both uniaxial tensions and compressions have a departure from the maximum shear stress direction $\left(45^{\circ}\right)$, then according to the MohrCoulomb criterion $[35,36]$, there is

$\tau_{0}=\tau_{\theta}+\alpha \sigma_{\theta}$.

where $\tau_{\theta}$ and $\sigma_{\theta}$ are the shear and normal stresses on the fracture plane, respectively, and could be derived from:

$\tau_{\theta}=\sigma_{F} \sin (\theta) \cos (\theta)$. 

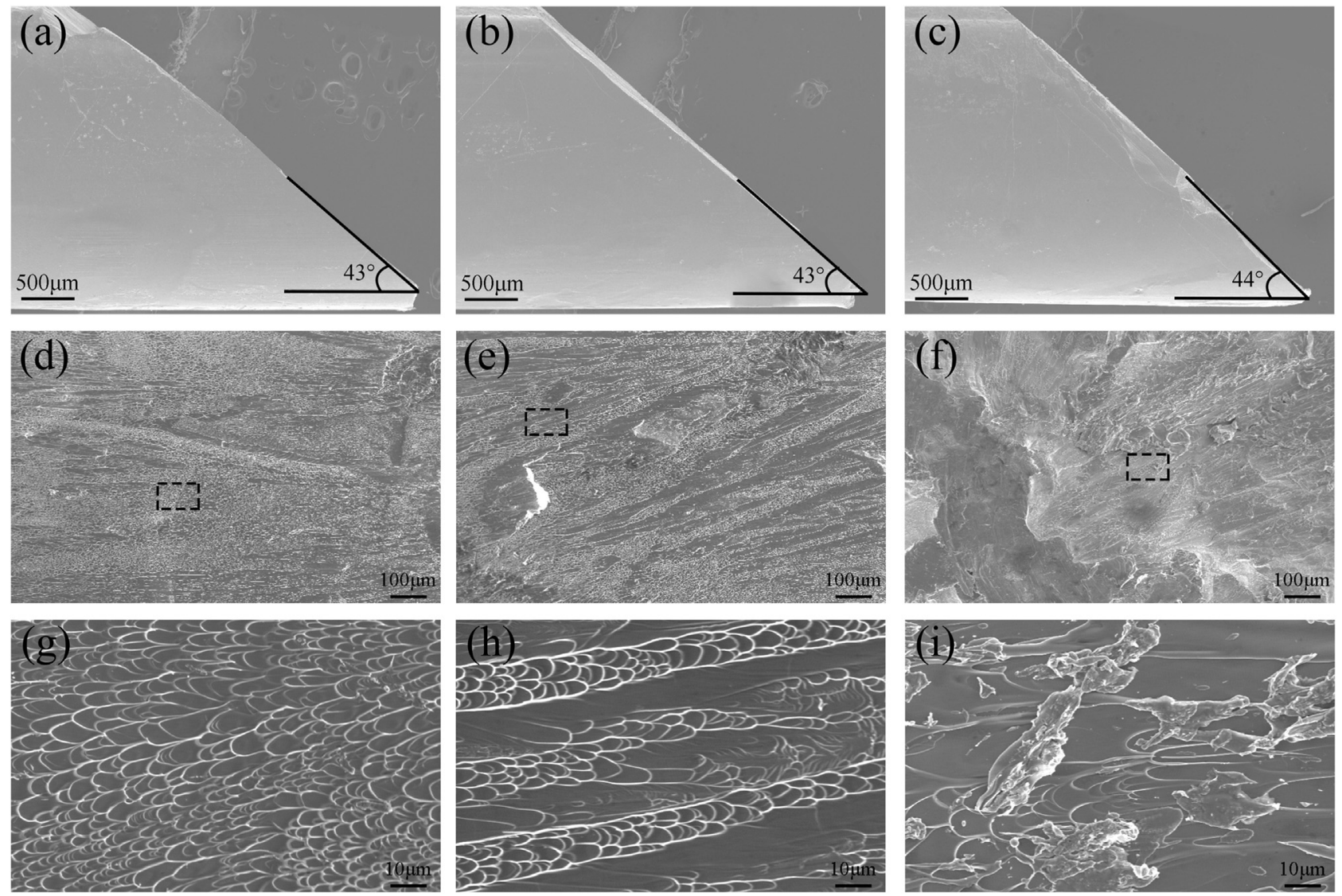

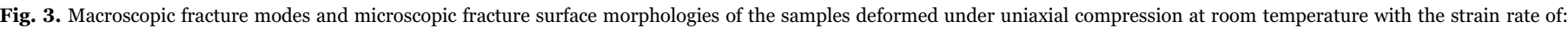
$2.0 \times 10^{-4} \mathrm{~s}^{-1}$ : (a), (d), (g); $1.0 \times 10^{-2} \mathrm{~s}^{-1}$ : (b), (e), (h); $3.2 \times 10^{3} \mathrm{~s}^{-1}:$ (c), (f), (i).

$\sigma_{\theta}=\sigma_{F} \sin ^{2}(\theta)$.

For uniaxial tensions, the fracture angles of all the samples are about $54^{\circ}$ (Fig. 2), then there is $\sin (\theta) \cos (\theta)=0.48$, which is very close to 0.5 . Therefore, it is reasonable to take the uniaxial tension yielding strength $\sigma_{y}$ as about twice as large as the shear yielding strength $\tau$. Consequently, the shear strain rate $\dot{\gamma}$ can be replaced by $2 \dot{\varepsilon}$. As for the uniaxial compressions, the fracture angles are about $43^{\circ}$ (Fig. 3), therefore, there is $\sin (\theta) \cos (\theta)=0.5$. In this regard, the approximate relationship between uniaxial tension yielding strength and the shear yielding strength is also suitable for the uniaxial compressive cases. In other words, for both uniaxial tension and compression, we have:

$\sigma_{y}=2 \tau_{y}$.

$\dot{\gamma}=2 \dot{\varepsilon}$

Substituting Eqs. (3), (7) and (8) into Eq. (1), there is:

$\frac{\sigma_{y}}{\sigma_{r}}=\frac{\tau_{0}}{\tau_{r}}-\frac{\tau_{0}}{\tau_{r}}\left[(k / \beta) \ln \left(\omega_{0} / 2 C \dot{\varepsilon}\right)\left(G_{0 T} / G_{0 T_{g}}\right)\right]^{2 / 3}\left[\left(T_{r}+\frac{\zeta \Omega \eta \dot{\varepsilon}^{2}}{2 \pi \kappa r_{0}}\right) / T_{g}\right]^{2 / 3}$.

where $\sigma_{r}=2 \tau_{r}$. is the quasi-static yielding strength at room temperature. $\tau_{r}$ and $\tau_{0}$ are the shear yielding strength at room and absolute zero temperature, respectively, with a relationship of: $\tau_{0}=\tau_{r} /\left[1-\left(0.2 T_{r} / T_{g}\right)^{2 / 3}\right][18]$.

With regard to Eq. (9), it should be noted that there is a critical value of the strain rate, which corresponds to the temperature rise in the shear band comes near to the glass transition temperature. In the current work, the highest strain rate, $3.2 \times 10^{3} \mathrm{~s}^{-1}$, is still lower than the critical value, which is about $6.0 \times 10^{3} \mathrm{~s}^{-1}$ or larger as pointed out by Liu and Liu [18]. Therefore, it can be accepted to neglect the precipitously decrease of the viscosity near the contiguous STZ. i.e., the viscosity can be taken as a constant. As for Zr-based BMGs, it is justifiable to take the parameters as the same to Ref [18]. Then one can take $\Omega=3.89 \times 10^{-27} \mathrm{~m}^{3}, \quad r_{0}=10^{-9} \mathrm{~m}, \quad \eta=10^{15} \mathrm{~Pa} \cdot \mathrm{s}, \quad \kappa=21 \mathrm{~W} /(\mathrm{m} \mathrm{K})$. According to Ref [30]., there is: $(k / \beta)\left(G_{0 T} / G_{0 T_{g}}\right)=0.0057$ and $\omega_{0} / 2 C=10^{13}$. For the current Vit $105 \mathrm{BMG}, T_{g}=690 \mathrm{~K}$, then there is $\tau_{0} / \tau_{r}=1.239$. By substituting all of the parameters into Eq. (9), we can obtain the relationship between the normalized yielding strength $\left(\sigma_{y} / \sigma_{r}\right)$ and the applied strain rate at room temperature, which is presented as the solid line in Fig. 4. It can be seen that the yielding strength has a weak dependence on the strain rate from $10^{-4} \mathrm{~s}^{-1}$ to about $4.5 \times 10^{2} \mathrm{~s}^{-1}$. However, there is an apparent decrease of the yielding strength when the strain rate increases to larger than

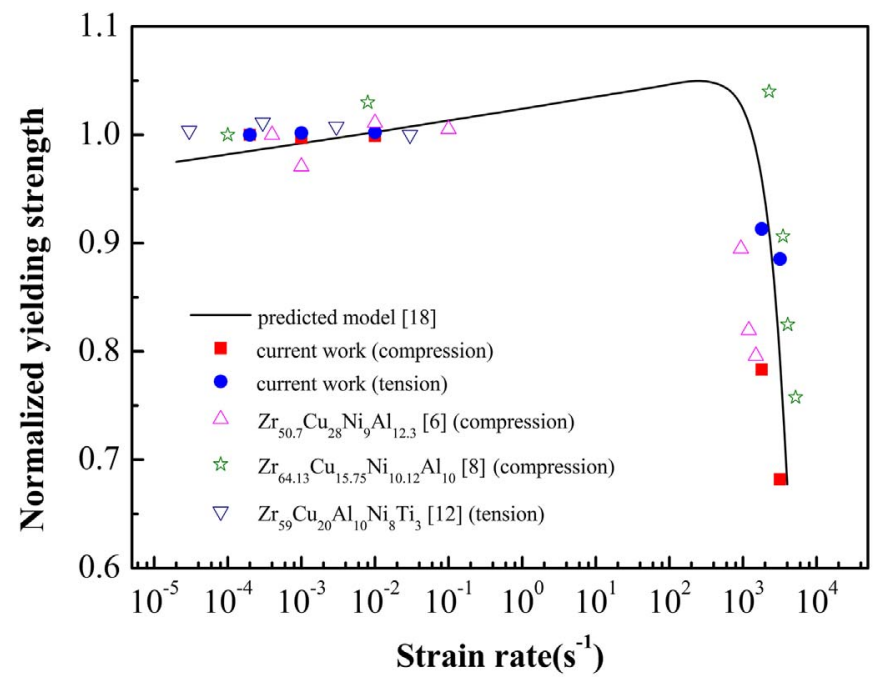

Fig. 4. The relationship between the normalized yielding strength and the strain rate. 
$4.5 \times 10^{2} \mathrm{~s}^{-1}$. i.e., there is a critical strain rate (about $4.5 \times 10^{2} \mathrm{~s}^{-1}$ ) for Vit $105 \mathrm{BMG}$, which corresponds to the transition of the strain rate effect of the yielding strength from insensitive to negative with increasing strain rate. If the tensile yielding strength under the lowest applied strain rate, $2.0 \times 10^{-4} \mathrm{~s}^{-1}$, is assigned to $\sigma_{r}$, then the variation of the normalized tensile yielding strength with the applied strain rate could be precisely obtained, which is shown in Fig. 4 as the solid dots. Similarly, the result of the uniaxial compression is also exhibited in Fig. 4 as the solid rectangles. In addition, some data from literatures $[6,8,12]$ are included in Fig. 4 as the hollow points. Obviously, the model could predict well the present and other experimental data that are obtained from both uniaxial tension and compression.

In the following, we try to understand the underlying mechanism in terms of the activation of the STZ, which is strongly influenced by local atomic rearrangements [30,37-39]. In a BMG specimen experiencing a uniform stress, it will be easier for the activation of STZ at the local regions with a higher free volume concentration [31,40-42]. When the strain rate varies from $10^{-4} \mathrm{~s}^{-1}$ to $10^{-2} \mathrm{~s}^{-1}$, the amount of the available free volume, which is defined as the free volume that can be mobile $[43,44]$, will be relatively large due to the sufficient time for the diffusion of the atoms and the rearrangements of the free volume. This is in good agreement with the regular fracture angles and the smooth fracture surfaces shown in Figs. 2 and 3. Therefore, the first STZ can be easily activated under a stress smaller than macro yielding strength. Then the surrounding material is subjected to a self-generated dynamic and thermal fluctuation. According to Eq. (2), the temperature rise under quasi-static strain rates is quite small, i.e., the thermal fluctuation result from the first STZ is very weak and can be neglected. This is consistent with the regular vein-like patterns and the less molten behavior under quasi-static strain rates as presented in Figs. 2 and 3. Consequently, the activation of the next STZ need a higher stress [45]. This process will repeat all the time until the advent of a shear band, which is due to the combination of a series of STZ, and finally results in the yielding of the macroscopic material. According to the above analysis, we can find that the existence of the available free volume will facilitate the activation of the STZ, indicating a softening effect on the yielding strength. At the same time, the STZs will occur more difficultly due to the fewer amount of available free volume under a higher strain rate, producing a strain rate hardening effect on the yielding strength. The combination of the free volume softening and the strain rate hardening finally results in the balance (or independence) of the yielding strength on the applied strain rate. When the strain rate increases to larger than a critical value (for Vit 105, it should be about $4.5 \times 10^{2} \mathrm{~s}^{-1}$ ), the free volume concentration will be larger with the increasing strain rate [46-48]. However, the available free volume will be fewer due to the shorter time for the diffusion of the atoms and the rearrangements of the free volume under a higher strain rate. According to the fracture angles and the corresponding fracture surface morphologies presented in Fig. 2. (2) and (3), we can see that the deformation of Vit 105 under dynamic cases is still governed by STZs $[3,44,49]$. In other words, there is still a free volume softening. At the same time, the fewer available free volume will hinder the activation of the first STZ, and then a larger stress is needed, producing a strain rate hardening. However, once the first STZ is activated, it will induce a considerable thermal effect, which will contribute to the rapid activation of the next STZ, the repetition of this process could produce a serious temperature rise around the STZs. This picture is in line with the more serious melting behavior on the dynamic fracture surfaces as presented in Figs. 2 and 3. The higher temperature will facilitate the quick activation and combination of the STZs, which will result in the rapid formation of a shear band and then incur the yielding of the macroscopic material. As a consequence, it is reasonable to conclude that there is a free volume softening, a strain rate hardening and a significant thermal softening under the dynamic cases. The combination of these three factors eventually results in the negative dependence of the yielding strength on the strain rate. In light of the above analysis, the initiation of the shear band under dynamic conditions is accompanied by a serious thermal softening, which will result in a significant decrease of material's viscosity in the shear band and then a reduction of the flow stress [50-52]. At the same time, the super-high loading rate allows almost no time for the structure recovery in the shear band. Consequently, there is a fast propagation of the shear band until the finally catastrophic failure. This is consistent with the stress-strain curves presented in Fig. 1b, i.e., the stress initially reaches a maximum and then there is a decline, which is in accordance with the findings reported by other researchers in $\mathrm{Zr}$-based BMGs [6,53]. As for the quasi-static cases, both the weakly thermal fluctuation and the enough time for the reconstruction of the material in the shear band contribute to the repeated activation and arrest of the shear band, which eventually results in the relatively large plastic flow as demonstrated in Fig. 1b.

It is interesting to note that the yielding strength under dynamic compression is lower than that under dynamic tension, which shows an opposite trend of the yielding strength difference between compression and tension under quasi-static condition. A similar behavior has been also revealed by Mukai et al. [10,54] who reported the strength data of a $\mathrm{Pd}_{40} \mathrm{Ni}_{40} \mathrm{P}_{20}$ bulk metallic glass in compression and tension over a wide strain rate range (from quasi-static to dynamic rates). Under the quasi-static strain rates, the yielding strength is mainly dominated by structural STZ operations. Due to its inherent dilatational nature [5558], the STZ occurs easier in tension than in compression, and thus the plastic yielding is achieved in the former with a relatively lower strength. With increasing strain rate into the dynamic range, an additional thermal softening should contribute to the plastic yielding that, although, is still governed by STZs. Obviously, dynamic compression should induce more significant thermal softening than dynamic tension. This is because, STZs in dynamic compression occur more difficultly and need higher temperature to activate. In our present work, we neglect the tension-compression asymmetry of structural STZ softening and thermal softening. However, such asymmetry changes with strain rates just provides solid evidence for the thermal softening that should be taken into account under dynamic rates. Nevertheless, the tension-compression asymmetry and its strain rate effect deserves to be studied detailedly in our future work.

\section{Conclusions}

The effect of strain rate on yielding strength of a $\mathrm{Zr}$-based bulk metallic glass is investigated by uniaxial tension and compression experiments over a wide range of applied strain rates at room temperature. The strain rate effect of the yielding strength has an apparent transition from insensitive to negative, which could be well described by the modified cooperative-shear model where the thermal effect and the associated free volume rearrangement are taken into account. The combination of free volume softening and strain rate hardening finally results in an insensitive strain rate dependence of the yielding strength under quasi-static conditions. When the strain rate increases above a critical value, there is a significant thermal softening in addition to the free volume softening and strain rate hardening. The coordination of these three factors eventually induces a negative strain rate sensitivity under dynamic cases. The present work is helpful to understand the relationship between the yielding strength and the strain rate in $\mathrm{Zr}$-based BMGs. Moreover, it will contribute to control the strain rate sensitivity by changing the relevant parameters.

\section{Acknowledgements}

The financial support from the National Natural Science Foundation of China (NSFC) under Grant nos. 51171138, 11522221, 11372315 and 51321003 are gratefully acknowledged. This work was also supported by a grant from Major Fundamental Research Projects of National Security (No. 613262). 


\section{References}

[1] Z.Q. Song, E. Ma, J. Xu, Acta Mater. 109 (2016) 275-285.

[2] T.C. Hufnagel, C.A. Schuh, M.L. Falk, Acta Mater. (2016) 375-393.

[3] F. Jiang, M.Q. Jiang, H.F. Wang, Y.L. Zhao, L. He, J. Sun, Acta Mater. 59 (2011) 2057-2068.

[4] W.F. Ma, H.C. Kou, J.S. Li, H. Chang, L. Zhou, J. Alloy. Compd. 472 (2009) $214-218$.

[5] J. Zhang, J.M. Park, D.H. Kim, H.S. Kim, Mater. Sci. Eng., A 449 (2006) 290-294.

[6] W. Zheng, Y.J. Huang, G.Y. Wang, P.K. Liaw, J. Shen, Metall. Mater. Trans. A 42 (2011) 1491-1498.

[7] H. Li, G. Subhash, X.-L. Gao, L.J. Kecskes, R.J. Dowding, Scr. Mater. 49 (2003) 1087-1092.

[8] W.D. Liu, K.X. Liu, X.X. Xia, W.H. Wang, J. Mater. Res. 25 (2010) 1230-1234.

[9] H.A. Bruck, A.J. Rosakis, W.L. Johnson, J. Mater. Res. 11 (1996) 503-511.

[10] T. Mukai, T.G. Nieh, Y. Kawamura, A. Inoue, K. Higashi, Scr. Mater. 46 (2002) $43-47$

[11] H. Tokunaga, K. Fujita, Y. Yokoyama, Mater. Trans. 53 (2012) 1395-1399.

[12] Z.F. Zhang, E. Jürgen, L. Schultz, Acta Mater. 51 (2003) 1167-1179.

[13] A. Bhattacharyya, G. Singh, K.E. Prasad, R. Narasimhan, U. Ramamurty, Mater. Sci. Eng. A 625 (2015) 245-251.

[14] D. Pan, A. Inoue, T. Sakurai, M.W. Chen, Proc. Natl. Acad. Sci. USA 105 (2008) $14769-14772$.

[15] J. Sort, J. Fornell, W. Li, S. Surinach, M. Baro, J. Mater. Res. 24 (2009) 918-925.

[16] Y. Ma, J.H. Ye, G.J. Peng, D.H. Wen, T.H. Zhang, Mater. Sci. Eng., A 627 (2015) $153-160$.

[17] T. Burgess, K.J. Laws, M. Ferry, Acta Mater. 56 (2008) 4829-4835.

[18] W.D. Liu, K.X. Liu, Appl. Phys. Lett. 100 (2012) 1419041-1419044.

[19] M.Q. Jiang, G. Wilde, J.H. Chen, C.B. Qu, S.Y. Fu, F. Jiang, L.H. Dai, Acta Mater. 77 (2014) 248-257.

[20] Z.F. Zhang, J. Eckert, Phys. Rev. Lett. 94 (2005) 0943011-0943014.

[21] Y. Chen, M.Q. Jiang, Y.J. Wei, L.H. Dai, Philos. Mag. 91 (2011) 4536-4554.

[22] R.T. Qu, J. Eckert, Z.F. Zhang, J. Appl. Phys. 109 (2011) $08354401-08354412$.

[23] R. Huang, Z. Suo, J.H. Prevost, W.D. Nix, J. Mech. Phys. Solids 50 (2002) 1011-1027.

[24] R.D. Conner, W.L. Johnson, N.E. Paton, W.D. Nix, J. Appl. Phys. 94 (2003) 904-911.

[25] P.S. Steif, F. Spaepen, J.W. Hutchinson, Acta Metall. 30 (1982) 447-455.

[26] X.J. Gu, S.J. Poon, G.J. Shiflet, J.J. Lewandowski, Acta Mater. 58 (2010) $1708-1720$.
[27] R.T. Qu, Z.Q. Liu, G. Wang, Z.F. Zhang, Acta Mater. 91 (2015) 19-33.

[28] R.T. Qu, Z.F. Zhang, J. Appl. Phys. 114 (2013) 19350401-19350411.

[29] A.L. Greer, Y.Q. Cheng, E. Ma, Mater. Sci. Eng. R 74 (2013) 71-132.

[30] W.L. Johnson, K. Samwer, Phys. Rev. Lett. 95 (2005) 195501-195504.

[31] A.S. Argon, Acta Metall. 27 (1979) 47-58.

[32] Z.Y. Liu, Y. Yang, C.T. Liu, Acta Mater. 61 (2013) 5928-5936.

[33] M.D. Demetriou, J.S. Harmon, M. Tao, D. Gang, K. Samwer, W.L. Johnson, Phys, Rev. Lett. 97 (2006) 0655021-0655024.

[34] M.D. Demetriou, W.L. Johnson, K. Samwer, Appl. Phys. Lett. 94 (2009) 191905.

[35] C.A. Schuh, A.C. Lund, Nat. Mater. 2 (2003) 449-452.

[36] J. Caris, J.J. Lewandowski, Acta Mater. 58 (2010) 1026-1036.

[37] D. Srolovitz, K. Maeda, V. Vitek, T. Egami, Philos. Mag. A 44 (1981) 847-866.

[38] D. Srolovitz, V. Vitek, T. Egami, Acta Metall. 31 (1983) 335-352.

[39] S.G. Mayr, Phys. Rev. Lett. 97 (2006) 195501-195504.

[40] F. Spaepen, Acta Metall. 25 (1977) 407-415.

[41] A.S. Argon, H.Y. Kuo, Mater. Sci. Eng. 39 (1979) 101-109.

[42] A.S. Argon, L.T. Shi, Philos. Mag. A 46 (1982) 275-294.

[43] M.Q. Jiang, G. Wilde, F. Jiang, L.H. Dai, Theor. Appl. Mech. Lett. (5) (2015) 200-204.

[44] M.C. Li, M.Q. Jiang, G. Li, L. He, J. Sun, F. Jiang, Intermetallics 77 (2016) 34-40.

[45] S. Wang, Y.F. Ye, B.A. Sun, C.T. Liu, S.Q. Shi, Y. Yang, J. Mech. Phys. Solids 77 (2015) 70-85.

[46] L.F. Liu, L.H. Dai, Y.L. Bai, B.C. Wei, J. Non-Cryst. Solids 351 (2005) 3259-3270.

[47] P. De Hey, J. Sietsma, A. Van Den Beukel, Acta Mater. 46 (1998) 5873-5882.

[48] W. Zheng, Y.J. Huang, J. Shen, Mater. Sci. Eng. A 528 (2011) 6855-6859.

[49] G. Li, M.Q. Jiang, F. Jiang, L. He, J. Sun, Appl. Phys. Lett. 102 (2013) 1719011-1719014.

[50] D. Tönnies, K. Samwer, P.M. Derlet, C.A. Volkert, R. Maaß, Appl. Phys. Lett. 106 (2015) 171907.

[51] R. Maaß, D. Klaumünzer, J.F. Löffler, Acta Mater. 59 (2011) 3205-3213.

[52] A.J. Cao, Y.Q. Cheng, E. Ma, Acta Mater. 57 (2009) 5146-5155.

[53] Y.F. Xue, H.N. Cai, L. Wang, F.C. Wang, H.F. Zhang, Mater. Sci. Eng. A 473 (2008) $105-110$.

[54] T. Mukai, T.G. Nieh, Y. Kawamura, A. Inoue, K. Higashi, Intermetallics 10 (2002) 1071-1077.

[55] A.S. Argon, Acta Mater. 27 (1979) 47-58.

[56] M.Q. Jiang, G. Wilde, L.H. Dai, Mech. Mater. 81 (2015) 72-83.

[57] Y.J. Wang, M.Q. Jiang, Z.L. Tian, L.H. Dai, Scr. Mater. 112 (2016) 37-41.

[58] M.Q. Jiang, G. Wilde, L.H. Dai, Scr. Mater. 127 (2017) 54-57. 